Perspective

\title{
Good practice guide for papers on supercapacitors and related hybrid capacitors for the Journal of Power Sources
}

\author{
Catia Arbizzani ${ }^{\mathrm{a}, *}$, Yan $\mathrm{Yu}^{\mathrm{b}}$, Jie $\mathrm{Li}^{\mathrm{c}}$, Jie Xiao ${ }^{\mathrm{d}}$, Yong-yao Xia ${ }^{\mathrm{e}}$, Yong Yang ${ }^{\mathrm{f}}$, Clara Santato ${ }^{\mathrm{g}}$, \\ Rinaldo Raccichini ${ }^{\mathrm{h}}$, Stefano Passerini ${ }^{\mathrm{i}, \mathrm{j}}$
}

a Department of Chemistry "Giacomo Ciamician", Alma Mater Studiorum Università di Bologna, Via F. Selmi 2, 40126, Bologna, Italy

${ }^{\mathrm{b}}$ University of Science and Technology of China (USTC), Department of Material Science \& Engineering, Hefei, China

${ }^{\mathrm{c}}$ Forschungszentrum Jülich GmbH, Helmholtz-Institute Münster, Münster, North Rhine-Westphalia, Germany

d Pacific Northwest National Laboratory, Seattle, WA, United States

e Department of Chemistry, Institute of New Energy, Fudan University, Shanghai, China

${ }^{\mathrm{f}}$ State Key Laboratory for Physical Chemistry of Solid Surfaces, Department of Chemistry, Xiamen University, Xiamen, China

${ }^{g}$ Department of Engineering Physics, École Polytechnique de Montreal, Montreal, Quebec, Canada

${ }^{\mathrm{h}}$ National Physical Laboratory, Hampton Road, Teddington, TW11 OLW, UK

${ }^{i}$ Helmholtz Institute Ulm, Electrochemistry I, Helmholtzstr. 11, 89081, Ulm, Germany

${ }^{\mathrm{j}}$ Karlsruhe Institute of Technology, P.O. Box 3640, 76021, Karlsruhe, Germany

\section{Brief introduction to supercapacitors}

The term supercapacitor (or ultracapacitor) [1] is used to indicate an electrochemical capacitor capable of storing charge through a capacitive process occurring in the electrical double layer formed at the interface between an electronic conductor (i.e., the electrode) and an electrolytic solution (e.g., non-aqueous electrolyte) [2,3]. The increasing demand for improved electrochemical energy storage systems continually boosts research efforts toward new materials, configurations and production processes, both for batteries and supercapacitors. The development of hybrid devices (e.g., where one electrode stores charge through a faradaic process and the other through a non-faradaic process) has also exploited progress in both the battery and supercapacitor fields. However, not all investigated materials are promising or industry-relevant, contrary to the claims in many research works. For example, complications related to the upscaling of lab-scale experiments hamper the exploitation at industrial scale. For these reasons, it is essential to define the best-practice methods to obtain reasonable predictions for supercapacitor materials and device testing. Also, many battery electrode materials are explored as supercapacitor electrode materials. However, not all battery materials can be considered as candidate electrode materials for hybrid devices. Only those materials with a suitable crystal structure for high rate capability and good cycling stability are appropriate.

\section{Good practice for the experimental section}

Given that the performance of individual electrodes and entire devices depends on the electrode mass loading [4,5], the following information must always be reported in the manuscript's experimental section:

1. Pore size distribution (ratio of mesopore volume to micropore volume) and surface area analysis of the active material.

2. Loading of active materials and total mass of the electrode, particularly for thin electrodes or if three-dimensional current collectors (sponge-like, foam, felts, etc.) are used.

3. Electrode area

4. Electrode thickness (when volumetric values are reported)

5. Composition and volume of the electrolyte

6. Type and number of separators

7. Potential range and/or current(s) applied

\section{Good practice for the results and discussion section}

To demonstrate the suitability of materials (or devices) for supercapacitor applications, it is necessary to provide the following specification in the manuscript main text:

1. Coulombic efficiency (which is essential for a long cycle-life)

2. Cycle number (preferably galvanostatic charge/discharge cycles rather than voltammetric cycles)

3. When comparative data are given (Tables or Ragone plot), please specify if the data refer to similar mass loadings (in Tables, the loading should be included). See also the example table in the section 6 of this document.

\footnotetext{
* Corresponding author.

E-mail address: catia.arbizzani@unibo.it (C. Arbizzani).
} 


\subsection{Figures}

1. Use the IUPAC or the American conventions (not mixed conventions) for voltammograms [6].

2. Nyquist plots of electrochemical impedance spectroscopy (-Im(Z) vs $\operatorname{Re}(\mathrm{Z}), \mathrm{Z}$ in $\Omega \mathrm{cm}^{2}$ ) should be ortho-normed (same scale for impedance on both ordinate and abscissa axes). The high and low frequency values should be stated, if different from those indicated in the Experimental section, in which the number of data points (per decade of frequency or overall) and the amplitude of the signal and direction of the sweep should be provided. Also, the operating conditions of the measurements should be given.

\section{Valid formulas for the evaluation of materials and devices}

The main problem in the use of the appropriate formulas for capacitance, energy and power evaluation is mainly linked to the concepts (and misconceptions) of capacitance and pseudocapacitance. Considering that many papers are available on this topic [7-9], here we summarise only a general outline with the most important key concepts.

Several types of active materials can be considered for supercapacitors: (i) those exhibiting capacitive behaviors like porous activated carbon, (ii) those exhibiting pseudocapacitive behaviors like $\mathrm{RuO}_{2}$ and $\mathrm{MnO}_{2}$, and (iii) those exhibiting faradaic, battery-like behaviors like $\mathrm{Ni}$ and Co hydroxides. The main difference between the last two types of material is that pseudocapacitive materials exhibit a voltage response deriving from faradaic electrode processes but displaying an overall linear dependence of the charge variation with potential (or voltage):

$\Delta Q(\mathrm{C})=C(\mathrm{~F}) * \Delta V(\mathrm{~V})$

The capacitance can thus be evaluated by the derivative $d(\Delta Q) / d$ $(\Delta V)$ [2]. The typical nearly rectangular cyclic voltammograms and linear potential profiles during both galvanostatic charge and discharge are typical features of capacitive and "true" pseudocapacitive materials [9].

The capacity of battery-like materials is given by the product of the number of moles of electrons passed in the faradaic process, and the Faraday constant:

$Q(\mathrm{C})=n(\mathrm{~mol}) * F\left(\mathrm{C} \mathrm{mol}^{-1}\right)$

These materials do not show a constant capacitance over the whole potential window. For this reason, it is better to compare capacity rather than capacitance $[9,10]$.

All the materials mentioned above can be coupled in a device: two capacitive electrodes will give an electrochemical double layer capacitor, two pseudocapacitive electrodes will give a pseudocapacitor. A battery-like electrode and a pseudocapacitive or capacitive electrode could be assembled in a hybrid supercapacitor, which can display an overall capacitive behavior. If one of the electrodes is made of an insertion material, such as those used in Li-ion or Na-ion batteries, the device will be a Li-ion capacitor or a Na-ion capacitor, and the capacity should be evaluated rather than the capacitance.

Several published papers report the correct formulas for the calculation of single-electrode capacitance, and full device capacitance, energy and power $[4,8,11-13]$.

In the case of a hybrid device with nearly capacitive behavior, it is possible to evaluate the energy storage capacity by integration of the discharge curve (eq. (3)) and then to calculate the system capacitance by using the energy-capacitance relation (eq. (4)) $[12,14,15]$.

$E_{\text {int }}=I \int_{t(V \min )}^{t(V \max )} U(t) d t$

$C=\frac{2 E_{\text {int }}}{V_{\max }^{2}}$
It should be pointed out that it is meaningless to quote the energy and power of a single electrode [16].

In addition, some materials (pseudocapacitors) display high capacitances, but their redox potential makes it difficult to couple with other electrode materials to make a realistic capacitor. Authors may need to give some examples of counter electrodes that can be combined with a proposed electrode material within a reasonable electrochemical window. In addition to 3-electrode testing, we recommend also including measurements in 2-electrode configuration.

For the evaluation of tests and performance of commercial devices, we recommend referring to Ref. [17].

\section{What are the most important properties for a supercapacitor?}

When "good" material properties for supercapacitors are claimed, it is important to indicate the application for which they are envisioned. According to the application, different metrics should be used.

Specific, areal and volumetric performances depend on the size of the final device and the application. For macro-devices (from portable size to large-size), the capacitance can be expressed as $\mathrm{F} \mathrm{g}^{-1}, \mathrm{~F} \mathrm{~cm}^{-2}, \mathrm{~F}$ $\mathrm{cm}^{-3}$. For micro-devices (e.g., electronics) and nano-devices (e.g., onchip, implantable devices), areal and volumetric parameters become of paramount importance. In these cases, the amount of active material could be very low, thus resulting in apparently impressive specific capacitance; the areal and volumetric parameters will give a more meaningful prediction, however. In the case of flexible devices, bending properties should also be reported.

The cycle number depends on the type of material and the envisioned use. For exploratory studies on "very new" materials, a number of cycles in the range 1000 and 5000 cycles is required, but for practical applications at least 20000 cycles are required. For metal-ion capacitors, at least 1000 cycles are recommended for material and device studies.

The quotation marks used to highlight the term "very new" indicate that the material should be adequately assessed depending on the context. For instance, in the past decades, much effort has been devoted to the production of novel carbon-based materials with innumerable shapes and forms, obtained from countless bio-organic sources such as seed, leaf, wood, husk, shell, or peel. However, for possible consideration by the present journal, the "novelty" of such materials should not be only related to the originality of the material production process (for this purpose, materials journals are considered more appropriate) but also to the electrochemical energy storage performance. For example, if the vastness of the plant kingdom is considered, there will always be a first time for a "novel carbon" obtained by plant leaf pyrolysis. From this point of view, the Journal of Power Sources is no longer interested in the shape or source of a given material by itself, and does not consider it as a novelty feature, unless a real advantage in terms of performance, cost or sustainability is clearly demonstrated.

\section{Reference values from literature and commercial devices}

When a comparison of material/device performance with existing literature data is included in the paper, particular attention should be paid to the coherence of the data. Specifically, only materials/devices with similar mass loading should be compared.

As an example of proper reporting, Table 1 contains a limited number of results available from the literature [13,18-20], and could be used as a guide for authors to present benchmark information for different device types.

\section{General remarks}

The Elsevier website states the following under the Duties of Authors regarding Originality and Acknowledgement of Sources:

"The authors should ensure that they have written entirely original works, and if the authors have used the work and/or words of others, 
Table 1

Example of parameters useful for comparison of electrode materials in various cell configurations.

\begin{tabular}{|c|c|c|c|c|c|c|c|}
\hline Capacitor device type & Electrolyte & $\begin{array}{l}\text { Electrode mass loading } \\
\left(\mathrm{mg} \mathrm{cm}^{-2}\right)\end{array}$ & $\begin{array}{l}C_{\text {active material }}(\mathrm{F} \\
\left.\mathrm{g}^{-1}\right)\end{array}$ & $\begin{array}{l}\text { Qactive material } \\
\left(\mathrm{mAh} \mathrm{g}^{-1}\right)\end{array}$ & $\begin{array}{l}\text { Capacitance/capacity retention } \\
\text { of the device }\end{array}$ & $\begin{array}{l}E_{\text {device }}(\mathrm{Wh} \\
\left.\mathrm{kg}^{-1}\right)\end{array}$ & $\begin{array}{l}P_{\text {device }}(\mathrm{kW} \\
\left.\mathrm{kg}^{-1}\right)\end{array}$ \\
\hline $\begin{array}{l}\text { Electrochemical double } \\
\text { layer }\end{array}$ & Aqueous & $\geq 2$ & $200-500$ & not relevant & $>90 \%$ after $>10 \mathrm{k}$ cycles & $\sim 10$ & $\sim 100$ \\
\hline $\begin{array}{l}\text { Electrochemical double } \\
\text { layer }\end{array}$ & Organic & $\geq 2$ & $100-300$ & not relevant & $>90 \%$ after $>10 \mathrm{k}$ cycles & $\sim 10$ & $\sim 100$ \\
\hline Pseudo & Aqueous & $\geq 2$ & $500-1500$ & not relevant & $>90 \%$ after $>1 \mathrm{k}$ cycles & $10-30$ & $10-50$ \\
\hline Pseudo & Organic & $\geq 2$ & $135-300$ & not relevant & $>90 \%$ after $>1 \mathrm{k}$ cycles & $2-40$ & $1-10$ \\
\hline $\begin{array}{l}\text { Hybrid/battery-like/ } \\
\text { metal-ion }\end{array}$ & Aqueous & $\geq 2$ & $50-200$ & $50-120$ & $>90 \%$ after $>1 \mathrm{k}$ cycles & $15-60$ & $5-50$ \\
\hline $\begin{array}{l}\text { Hybrid/battery-like/ } \\
\text { metal-ion }\end{array}$ & Organic & $\geq 2$ & $30-100$ & $30-80$ & $>90 \%$ after $>1 \mathrm{k}$ cycles & $20-150$ & $5-50$ \\
\hline
\end{tabular}

that this has been appropriately cited or quoted and permission has been obtained where necessary. Proper acknowledgement of the work of others must always be given. Authors should cite publications that have influenced the reported work and that give the work appropriate context within the larger scholarly record. Information obtained privately, as in conversation, correspondence, or discussion with third parties, must not be used or reported without explicit, written permission from the source. Plagiarism takes many forms, from 'passing off' another's paper as the author's own paper, to copying or paraphrasing substantial parts of another's paper (without attribution), to claiming results from research conducted by others. Plagiarism in all its forms constitutes unethical behavior and is unacceptable."

For further details please see: https://www.elsevier.com/about/poli cies/publishing-ethics.

Highlights consist of a small number of bullet points that concisely capture the novel results of your research as well as any new methods that were used during the study.

The graphs and figures should exhibit a consistent and homogeneous presentation; color codes and font sizes should be maintained throughout to enable good legibility of the experimental data.

The use of emphatic statements such as "superior", "excellent", "outstanding" should be limited. The performance of tested materials/ components should be benchmarked versus state-of-the-art materials/ components and compared to the relevant literature.

\section{References}

[1] J.R. Miller, A.F. Burke, Electrochemical capacitors: challenges and opportunities for real-world applications, Electrochem. Soc. Interface 17 (2008) 53-57.

[2] B.E. Conway, Electrochemical Supercapacitors: Scientific Fundamentals and Technological Applications, Springer, 1999.

[3] M. Winter, R.J. Brodd, What are batteries, fuel cells, and supercapacitors? Chem. Rev. 104 (2004) 4245-4269, https://doi.org/10.1021/cr020730k.

[4] M.D. Stoller, R.S. Ruoff, Best practice methods for determining an electrode material's performance for ultracapacitors, Energy Environ. Sci. 3 (2010) 1294-1301, https://doi.org/10.1039/c0ee00074d.

[5] Y. Gogotsi, P. Simon, True performance metrics in electrochemical energy storage, Science 334 (80) (2011) 917-918, https://doi.org/10.1126/science.1213003.

[6] C. Zoski, Handbook of Electrochemistry, Elsevier Science Ltd., 2006.
[7] T. Brousse, D. Belanger, J.W. Long, To Be or not to Be pseudocapacitive? J. Electrochem. Soc. 162 (2015) A5185-A5189, https://doi.org/10.1149/ 2.0201505jes.

[8] S. Zhang, N. Pan, Supercapacitors performance evaluation, Adv. Energy Mater. 5 (2015) 1401401, https://doi.org/10.1002/aenm.201401401.

[9] M.R. Lukatskaya, B. Dunn, Y. Gogotsi, Multidimensional materials and device architectures for future hybrid energy storage, Nat. Commun. 7 (2016) 12647, https://doi.org/10.1038/ncomms12647.

[10] A.L. Brisse, P. Stevens, G. Toussaint, O. Crosnier, T. Brousse, $(\mathrm{OH} \mathrm{Ni,} 2$ and $\mathrm{NiO}$ based composites: battery type electrode materials for hybrid supercapacitor devices, Materials (Basel) 11 (2018) 1178, https://doi.org/10.3390/ma11071178.

[11] A. Balducci, D. Belanger, T. Brousse, J.W. Long, W. Sugimoto, Perspective-a guideline for reporting performance metrics with electrochemical capacitors: from electrode materials to full devices, J. Electrochem. Soc. 164 (2017) A1487-A1488, https://doi.org/10.1149/2.0851707jes.

[12] A. Laheäär, P. Przygocki, Q. Abbas, F. Béguin, Appropriate methods for evaluating the efficiency and capacitive behavior of different types of supercapacitors, Electrochem. Commun. 60 (2015) 21-25, https://doi.org/10.1016/j. elecom.2015.07.022.

[13] A. Noori, M.F. El-Kady, M.S. Rahmanifar, R.B. Kaner, M.F. Mousavi, Towards establishing standard performance metrics for batteries, supercapacitors and beyond, Chem. Soc. Rev. 48 (2019) 1272-1341, https://doi.org/10.1039/ c8cs00581h.

[14] A. Krause, P. Kossyrev, M. Oljaca, S. Passerini, M. Winter, A. Balducci, Electrochemical double layer capacitor and lithium-ion capacitor based on carbon black, J. Power Sources 196 (2011) 8836-8842, https://doi.org/10.1016/j. jpowsour.2011.06.019.

[15] S. Dsoke, B. Fuchs, E. Gucciardi, M. Wohlfahrt-Mehrens, The importance of the electrode mass ratio in a Li-ion capacitor based on activated carbon and Li4Ti5O12, J. Power Sources 282 (2015) 385-393, https://doi.org/10.1016/j. jpowsour.2015.02.079.

[16] M.N. Obrovac, V.L. Chevrier, Alloy negative electrodes for Li-ion batteries, Chem. Rev. 114 (2014) 11444-11502, https://doi.org/10.1021/cr500207g.

[17] A. Burke, M. Miller, Testing of electrochemical capacitors: capacitance, resistance, energy density, and power capability, Electrochim. Acta 55 (2010) 7538-7548, https://doi.org/10.1016/j.electacta.2010.04.074.

[18] J. Ding, W. Hu, E. Paek, D. Mitlin, Review of hybrid ion capacitors: from aqueous to lithium to sodium, Chem. Rev. 118 (2018) 6457-6498, https://doi.org/10.1021/ acs.chemrev.8b00116.

[19] P. Simon, T. Brousse, F. Favier, Supercapacitors Based on Carbon or Pseudocapacitive Materials, Wiley, 2017.

[20] A. Varzi, C. Schütter, J. Krummacher, R. Raccichini, C. Wolff, G.T. Kim, S. Rösler, B. Blumenröder, T. Schubert, S. Passerini, A. Balducci, A 4 Farad high energy electrochemical double layer capacitor prototype operating at $3.2 \mathrm{~V}$ (IES prototype), J. Power Sources 326 (2016) 162-169, https://doi.org/10.1016/j. jpowsour.2016.06.123. 